\title{
AN EXPANSION OF FREQUENCY DETERMINANTS WITH APPLICATION TO THE NORMAL FREQUENCIES OF A SPRING MOUNTED RIGID BODY (RESILIENT FOUNDATION)*
}

By F. K. G. ODQVIST (Royal Institute of Technology, Stockholm

1. Introduction. The free small oscillations of a conservative system of $n$ masses $Q_{i}$ with the coordinates $q_{i}$ and velocities $\dot{q}_{i}$ are governed by Lagrange's equations of the type

$$
\frac{d}{d t}\left(\frac{\partial T}{\partial \dot{g}_{i}}\right)+\frac{\partial W}{\partial q_{i}}=0, \quad i=1, \cdots, n,
$$

where the kinetic energy is

and the potential energy is

$$
T=\frac{1}{2} \sum_{i} Q_{i} \dot{q}_{i}^{2}
$$

$$
W=\frac{11}{2} \sum_{i, j} k_{i j} q_{i} q_{i} .
$$

In the sequel we shall limit the treatment to the case where the masses $Q_{i}$ and the spring constants $k_{i j}$ are constants in space and time.

The solution of (1.1) may be put in the form

$$
q_{i}=A_{i} e^{i \omega t}, \quad i=1, \cdots . n,
$$

where $A_{i}$ is a constant amplitude, and $\omega$ is the angular frequency the eigenvalues of which may be obtained from the equation

where

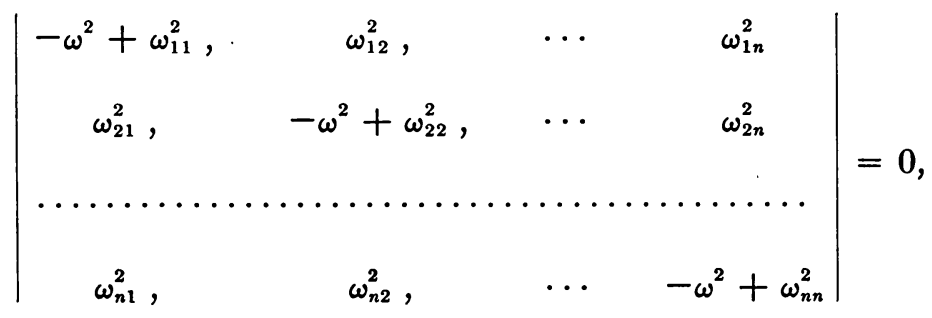

$$
\frac{k_{i i}}{Q_{i}}=\omega_{i i}^{2} .
$$

There are also other mechanical systems leading to the same type of frequency equation (1.3), e.g. the free vibrations of a rigid body, supported on elastic springs, if the coordinate system is properly chosen. In this case we have $n \leq 6$.

2. Expansion of frequency determinants. A frequency or "secular" determinant of the form

$$
\Delta(x)=\left|\begin{array}{cccc}
a_{1}+x, & a_{12}, & \ldots & a_{1 n} \\
a_{21}, & a_{2}+x, & \ldots & a_{2 n} \\
\ldots \ldots \ldots \ldots \ldots \ldots \ldots \ldots \ldots \ldots & \ldots \ldots \ldots
\end{array}\right|
$$

*Received April 23, 1951. 
may be developed in the series

$$
\begin{aligned}
\Delta(x)=\prod_{i}^{n}\left(a_{i}+x\right) & +\sum_{i<k}^{n}\left|\begin{array}{cc}
0, & a_{i k} \\
a_{k j}, & 0
\end{array}\right| \prod_{i}^{n}(i, k)\left(a_{i}+x\right) \\
& +\sum_{i<k<l}^{n}\left|\begin{array}{ccc}
0, & a_{i k}, & a_{i l} \\
a_{k i}, & 0, & a_{k l} \\
a_{l i}, & a_{l k}, & 0
\end{array}\right| \prod^{(i, k, l)}\left(a_{i}+x\right)+\cdots
\end{aligned}
$$$$
+\sum_{i<i}^{n}\left|\begin{array}{cccc}
0, & a_{12}, & \ldots & a_{1 n} \\
a_{21}, & 0, & . . & a_{2 n} \\
\ldots \ldots \ldots \ldots \ldots \ldots \ldots & \ldots \ldots \\
a_{n 1}, & a_{n 2}, & \ldots & 0
\end{array}\right|\left(a_{i}+x\right)\left(a_{i}+x\right)
$$$$
+\sum_{i}^{n}\left|\begin{array}{cccc}
0, & a_{12}, & \ldots & a_{1 n} \\
a_{21}, & 0, & \ldots & a_{2 n} \\
\ldots \ldots \ldots \ldots \ldots \ldots & \ldots \ldots
\end{array}\right|^{(i)}\left(a_{i}+x\right)
$$$$
+\left|\begin{array}{cccc}
0, & a_{12}, & \ldots & a_{1 n} \\
a_{21}, & 0, & \ldots & a_{2 n} \\
\ldots \ldots \ldots \ldots \ldots \ldots & \ldots \ldots \\
a_{n 1}, & a_{n 2}, & \ldots & 0
\end{array}\right|
$$

which may be obtained by induction or by considering $\Delta$ as function of $n$ variables $y_{i}=a_{i}+x$ and using McLaurins development for this function of $n$ variables.*

In (2.1) upper indices on a determinant indicate that the minor, obtained by suppressing the corresponding rows and columns, is to be taken; upper indices on a product sign indicate that the corresponding factors in the product are to be replaced by unity. It is of interest to note that products of the type

$$
\prod_{i}^{n}(i)\left(a_{i}+x\right)
$$

that is products of degree $n-1$ in $x$, do not occur in (2.1).

${ }^{*}$ The latter method was suggested to the author by Mr. L. E. Zachrisson. 
The expansion (2.1) may be used with advantage to approximate the roots of the determinantal equation (1.3).

3. The roots of the equation $\Delta(x)=0$. Under the assumption that the diagonal terms $a_{1}, \cdots a_{n}$ of the matrix are all different and absolutely: greater than the terms $a_{i i},(i \neq j)$ outside the diagonal*, it is easy to obtain approximate expressions for all the roots of the equation $\Delta(x)=0$.

To this effect we multiply all the terms $a_{i i}$ with a common factor $\lambda$, so that the expansion (2.1) takes the form of a polynomial of $n$ :th degree in $\lambda$, where the first degree term is missing.

Putting the $r$ :th root $x_{r}$ of the equation $\Delta(x)=0$ in the form of a power series in $\lambda$, and determining its coefficients by introducing this expression into the equation, we obtain

$$
x_{r}=-a_{r}+\lambda^{2} \frac{\sum_{i<k}^{n}\left|\begin{array}{rr}
0, & a_{i k} \\
a_{k i}, & 0
\end{array}\right| \prod_{i}^{n}\left(a^{\left(i, k^{\prime}, r\right)}\left(a_{i}-a_{r}\right)\right.}{\prod_{i}^{n}(r)\left(a_{i}-a_{r}\right)}+\lambda^{3}(\cdots), \quad(r=1, \cdots n)
$$

This power series in $\lambda$ will converge for sufficiently small $|\lambda|$, which means a condition for the magnitude of the matrix elements $\left|a_{i i}\right|$.

The condition, imposed at the outset, that all the diagonal terms $a_{i}$ be different and dominate the $a_{i j}$ is not essential.

In fact, it is easily seen that in the simple cases $n=2$ and 3 it will make no difference, if one of the diagonal terms be included among the small terms, e.g. if one of the terms $a_{i}$ be multiplied with $\lambda$ just as the terms $a_{i i}$ above. In these cases similar, though somewhat modified expansions for the roots $x_{r}$ will be possible. On the other hand it may be seen in the case $n=3$, that if $t w o$ of the diagonal terms, e.g. $a_{2}$ and $a_{3}$, be multiplied with $\lambda$, but not $a_{1}$, then an expansion of the type (3.1) will be possible for $x_{1}$ but not for $x_{2}$ and $x_{3}$, unless certain conditions be imposed upon the matrix.

We shall leave the question at this point and give an illustration of the treatment of a special case of practical importance in the next section.

\section{Application to spring mounted foundations.}

Spring mounted or resilient foundations for stationary machinery, prime movers etc. are built in a great variety of types and forms. In many cases, though, the design will be something like the one shown in Fig. 1, and the assumptions made below will hold, at least approximately. We shall consider the foundation as rigid and the springs to have spring constants according to Hooke's law. In this case there are in general six degrees of freedom of the system. Comparatively few references treat the problem with such generality [1]-[4], [6].†

If the coordinate system be chosen so that its axes coincide with the principal axes of inertia at the centre of gravity C.G. of the foundation, the frequency equation of the motion will have the form (1.3). We shall assume the axis C.G.-3 to be vertical-which is often true or nearly true- so that the axes C.G.-1 and C.G.-2 are to be found in a horizontal plane through C.G. Further we take $q_{1}, q_{2}, q_{3}$ to be translations of the founda-

\footnotetext{
*This assumption corresponds to what is generally termed a "weak coupling" of the system. $\dagger$ Numbers in brackets refer to the bibliography at the end of the paper.
} 
dation in directions of the coordinate axes and $q_{4} / r_{1}, q_{5} / r_{2}, q_{6} / r_{3}$ to be rotations with respect to the same axes, where $r_{1}, r_{2}, r_{3}$ are the corresponding radii of gyration of the foundation.

Furthermore, we assume the springs to have the following properties, often fulfilled in practice:
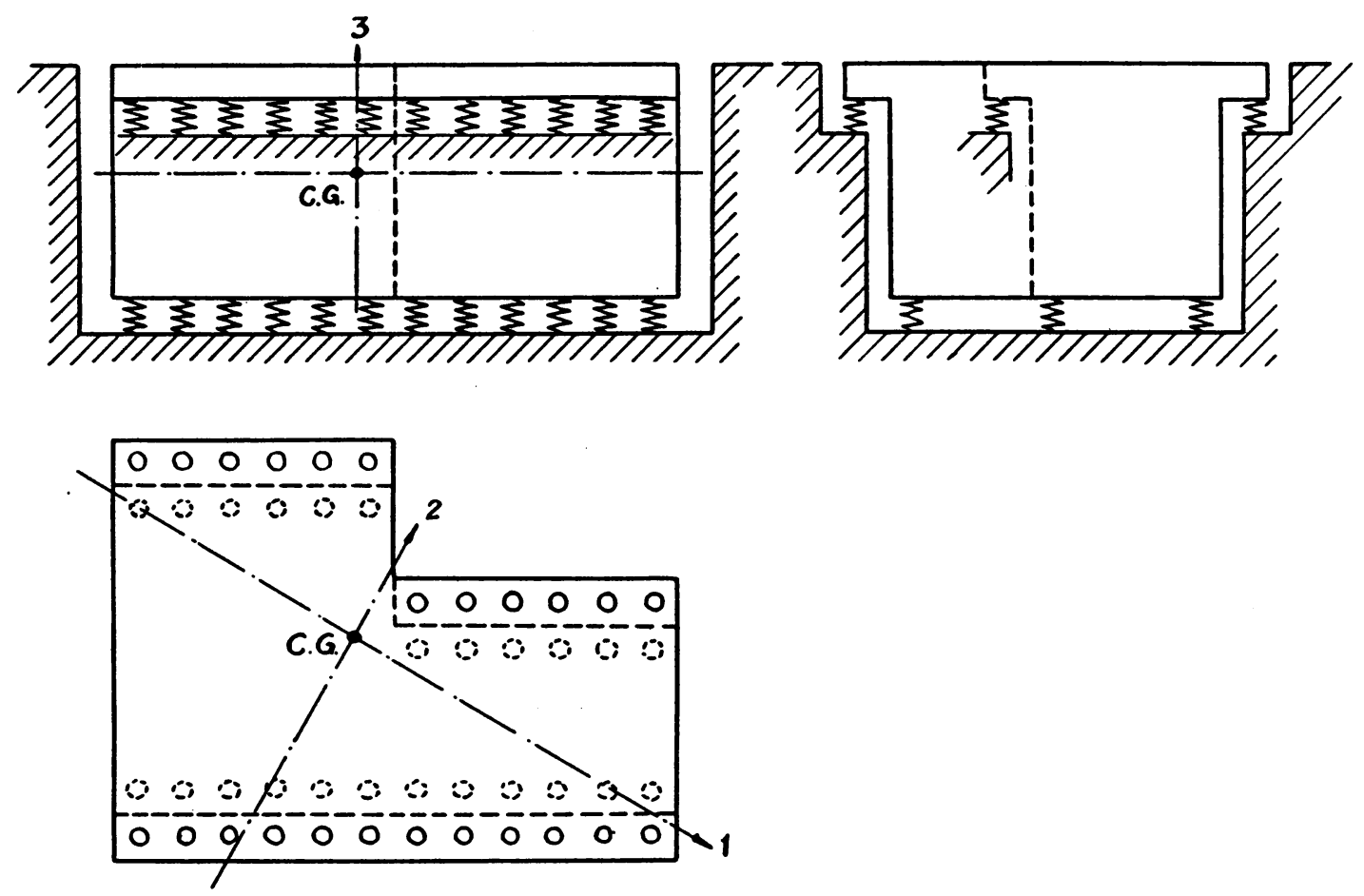

FIG. 1.

(a) all springs have vertical axes, e.g. are statically submitted to pure compression;

(b) each spring has a spring constant in vertical direction much larger than the one in a horizontal direction (for parallel displacements of the end faces of the springs). This will hold true f.i. for the ordinary type of rubber springs, used in this connection [5], [6], but it will generally not be the case for ordinary helical steel springs. Fig. 2 shows the relation between horizontal and vertical spring constant $k_{h}: k$, for different values of the relative compression $\xi$ for the ordinary type of cylindrical rubber springs, vulcanized to steel plates, according to [6].

(c) All springs have over-all dimensions small as compared with the foundation. If $h_{1 \nu}, h_{2 \nu}, h_{3 v}$ are the coordinates of the point of application of the $v$ :th spring and its spring constants in direction of the axes correspondingly $\kappa k_{1 v}, \kappa k_{2}, k_{3}$, where, according to (b), $\kappa$ may be taken as a small dimension-less quantity and $k_{1}, k_{2}$, and $k_{3}$, are all of the same order of magnitude, then the springs may be arranged by the designer so as to fulfill the conditions

$$
\sum_{V} k_{3 v} h_{2 v}=0, \quad \sum_{V} k_{3}, h_{1 v}=0, \quad \sum_{V} k_{3}, h_{1 v}, h_{2 v}=0
$$


the summations being extended over all the springs. From fig. 2 it is seen that $\kappa$ for rubber mountings, used in practice, will be of the order $0.1-0.15$ (shaded area).

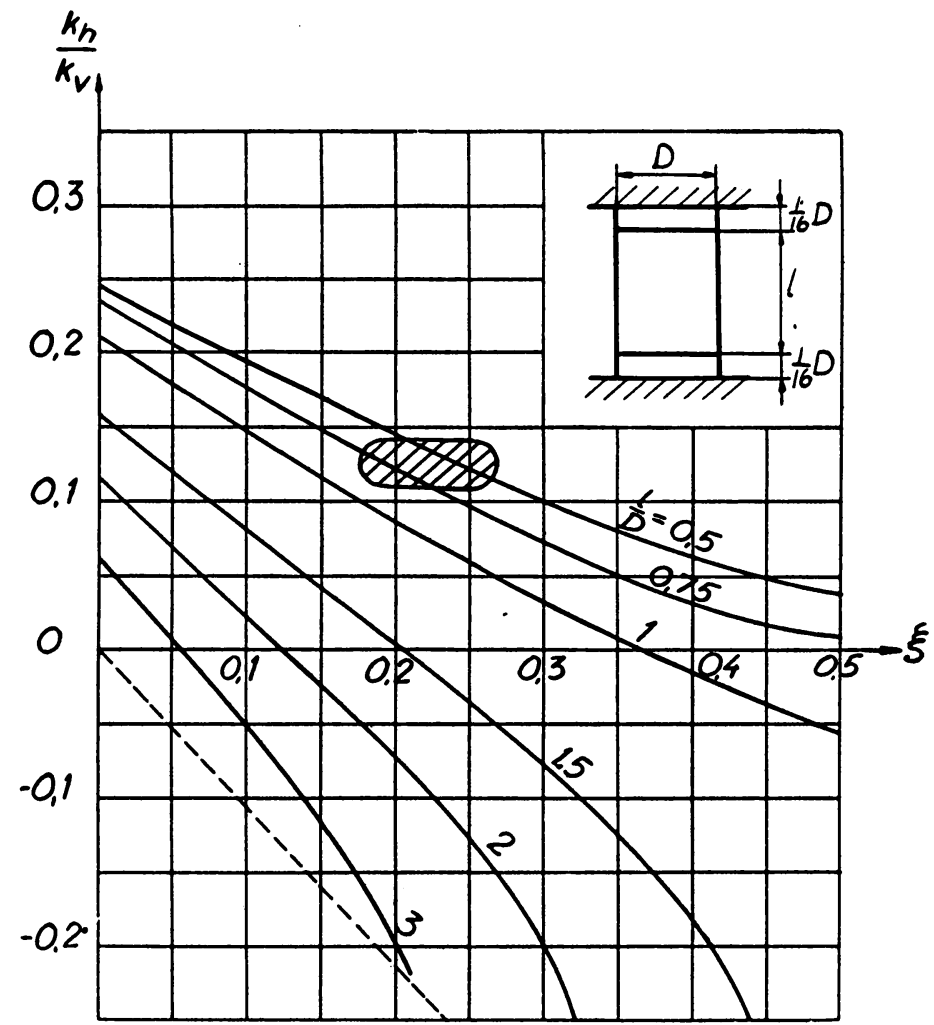

Fig. 2.

From (a), (b) and (c) it follows that the potential energy takes the simple form

$$
W=\frac{1}{2} \sum_{\nu}\left(\kappa k_{1}, \delta_{1}^{2},+\kappa k_{2}, \delta_{2 v}^{2}+k_{3}, \delta_{3 v}^{2}\right) \text {, }
$$

where

$$
\begin{aligned}
& \delta_{1 v}=q_{1}+q_{5} h_{3 v} / r_{2}-q_{6} h_{2 v} / r_{3}, \\
& \delta_{2 v}=q_{2}+q_{6} h_{1 v} / r_{3}-q_{4} h_{3 v} / r_{1}, \\
& \delta_{3 v}=q_{3}+q_{4} h_{2 v} / r_{1}-q_{5} h_{1 v} / r_{2} .
\end{aligned}
$$

The assumptions (a), (b), (c) make possible further considerable simplification of the frequency equation (1.3). Due to (c) we may write

$$
\omega_{i i}^{2}=\omega_{i i}^{* 2} \pm \kappa \bar{\omega}_{i i}^{2},
$$

where the upper or lower sign should be taken so as to make $\bar{\omega}_{i j}$ real. Then $\omega_{i i}^{*}$ and $\bar{\omega}_{i j}$ are of the same order of magnitude, if different from zero at all. 
Accordingly, we put ( $Q=$ total mass of foundation)

$$
\begin{aligned}
& \omega_{11}^{2}=\kappa \bar{\omega}_{11}^{2}=\frac{\kappa}{Q} \sum_{\nu} k_{1}, \quad \dot{\omega}_{15}^{\dot{2}}=\kappa \bar{\omega}_{15}^{2}=\frac{\kappa}{Q r_{2}} \sum_{\nu} k_{1 v} h_{3 \nu} \\
& \omega_{16}^{2}=-\kappa \bar{\omega}_{16}^{2}=-\frac{\kappa}{Q r_{3}} \sum_{\nu} k_{1}, h_{2 v}, \quad \omega_{22}^{2}=\kappa \bar{\omega}_{22}^{2}=\frac{\kappa}{Q} \sum_{\nu} k_{2 \nu}, \\
& \omega_{24}^{2}=-\kappa \bar{\omega}_{24}^{2}=-\frac{\kappa}{Q r_{1}} \sum_{\nu} k_{2 v} h_{3 v}, \quad \omega_{26}^{2}=\kappa \bar{\omega}_{26}^{2}=\frac{\kappa}{Q r_{3}} \sum_{\nu} k_{2 v} h_{1 \nu}, \\
& \omega_{33}^{2}=\frac{1}{Q} \sum_{\nu} k_{3 \nu}, \quad \omega_{42}^{2 !}=-\bar{\omega}_{42}^{2}=-\frac{\kappa}{Q r_{1}} \sum_{\nu} k_{2 \nu} h_{3 \nu} \\
& \omega_{44}^{2}=\omega_{44}^{* 2}+\kappa \bar{\omega}_{44}^{2}=\frac{1}{Q r_{1}^{2}} \sum_{\nu}\left(k_{3}, h_{2 \nu}^{2}+\kappa k_{2 \nu} h_{3 \nu}^{2}\right) \\
& \omega_{46}^{2}=-\kappa \bar{\omega}_{46}^{2}=-\frac{\kappa}{Q r_{1} r_{3}} \sum_{\nu} k_{2 v} h_{1 \nu} h_{3 \nu}, \quad \omega_{51}^{2}=\kappa \bar{\omega}_{51}^{2}=\frac{\kappa}{Q r_{2}} \sum_{\nu} k_{1 \nu} h_{3 \nu}, \\
& \omega_{56}^{2}=-\kappa \bar{\omega}_{56}^{2}=-\frac{\kappa}{Q r_{2} r_{3}} \sum_{\nu} k_{1 \nu} h_{2 \nu} h_{3 \nu} \\
& \omega_{55}^{2}=\omega_{55}^{* 2}+\kappa \bar{\omega}_{55}^{2}=\frac{1}{Q r_{2}^{2}} \sum_{\nu}\left(k_{3 \nu} h_{1 \nu}^{2}+\kappa k_{1 \nu} h_{3 \nu}^{2}\right), \\
& \omega_{61}^{2}=-\kappa \bar{\omega}_{61}^{2}=-\frac{\kappa}{Q r_{3}} \sum_{\nu} k_{1 \nu} h_{2 \nu}, \quad \omega_{62}^{2}=\kappa \bar{\omega}_{62}^{2}=\frac{\kappa}{Q r_{3}} \sum_{\nu} k_{2 \nu} h_{1 \nu}, \\
& \omega_{64}^{2}=-\kappa \bar{\omega}_{64}^{2}=-\frac{\kappa}{Q r_{1} r_{3}} \sum_{\nu} k_{2 \nu} h_{1 \nu} h_{3 \nu}, \quad \omega_{65}^{2}=-\kappa \bar{\omega}_{65}^{2}=-\frac{\kappa}{Q r_{2} r_{3}} \sum_{\nu} k_{1 \nu} h_{2 \nu} h_{3 \nu}, \\
& \omega_{66}^{2}=\kappa \bar{\omega}_{66}^{2}=\frac{\kappa}{Q r_{3}^{2}} \sum_{\nu}\left(k_{1 \nu} h_{2 \nu}^{2}+k_{2 \nu} h_{1 \nu}^{2}\right)
\end{aligned}
$$

All the rest of the quantities $\omega_{i j}^{2}$ vanish

$$
\omega_{12}^{2}=\omega_{13}^{2}=\omega_{14}^{2}=\omega_{21}^{2}=\omega_{23}^{2}=\omega_{25}^{2}=\omega_{31}^{2}=\omega_{32}^{2}=\omega_{36}^{2}=\omega_{41}^{2}=\omega_{52}^{2}=\omega_{63}^{2}=0 .
$$

for reasons of symmetry, and

$$
\omega_{34}^{2}=\omega_{35}^{2}=\omega_{43}^{2}=\omega_{45}^{2}=\omega_{53}^{2}=\omega_{54}^{2}=0
$$




$$
\begin{aligned}
& \Delta\left(-\omega^{2}\right)= \\
& \left|\begin{array}{cccccc}
-\omega^{2}+\kappa \bar{\omega}_{11}^{2}, & 0, & 0, & 0, & \kappa \bar{\omega}_{15}^{2}, & -\kappa \bar{\omega}_{16}^{2} \\
0, & -\omega^{2}+\kappa \bar{\omega}_{22}^{2}, & 0, & -\kappa \bar{\omega}_{24}^{2}, & 0, & \kappa \bar{\omega}_{26}^{2} \\
0, & 0, & -\omega^{2}+\omega_{33}^{2}, & 0, & 0, & 0 \\
0, & -\kappa \bar{\omega}_{42}^{2}, & 0, & -\omega^{2}+\omega_{44}^{* 2}+\kappa \bar{\omega}_{44}^{2}, & 0, & -\kappa \bar{\omega}_{46}^{2} \\
\kappa \bar{\omega}_{61}^{2}, & 0, & 0, & 0, & -\omega^{2}+\omega_{55}^{* 2}+\kappa \bar{\omega}_{55}^{2}, & -\kappa \bar{\omega}_{56}^{2} \\
-\kappa \bar{\omega}_{61}^{2}, & \kappa \bar{\omega}_{62}^{2}, & 0, & -\kappa \bar{\omega}_{64}^{2}, & -\kappa \bar{\omega}_{65}^{2}, & -\omega^{2}+\kappa \bar{\omega}_{68}^{2}
\end{array}\right|
\end{aligned}
$$

Due to the comparatively great number of small terms $\omega_{i i}^{2}(i=1,2,6)$ in the diagonal, it is not possible to obtain expansions in power series in $\kappa$ of the type (3.1) for the roots of the equation.

If, on the other hand, we submit the matrix to the additional conditions

$$
\bar{\omega}_{18}^{2}=\bar{\omega}_{61}^{2}=\bar{\omega}_{26}^{2}=\bar{\omega}_{\theta 2}^{2}=0,
$$

which are equivalent to the conditions

$$
\sum_{\nu} k_{1 v} h_{2 v}=0, \quad \sum_{\nu} k_{2 \nu} h_{1 v}=0,
$$

such developments will hold. It is to be noted that in many practical cases the conditions (4.7) will be a consequence of the two first conditions (4.1), due to the properties of the spring elements, such as disclosed by fig. 2 .

We may now put

$$
\begin{aligned}
& \omega_{1}^{2}=\kappa \bar{\omega}_{11}^{2}+\kappa^{2} \mu_{1}+\cdots, \quad \omega_{2}^{2}=\kappa \bar{\omega}_{22}^{2}+\kappa^{2} \mu_{2}+\cdots \\
& {\left[\omega_{3}^{2}=\omega_{33}^{2}\right], \quad \omega_{4}^{2}=\omega_{44}^{* 2}+\kappa \bar{\omega}_{44}^{2}+\kappa^{2} \mu_{4}+\cdots} \\
& \omega_{5}^{2}=\omega_{55}^{* 2}+\kappa \bar{\omega}_{55}^{2}+\kappa^{2} \mu_{5}+\cdots, \quad \omega_{6}^{2}=\kappa \bar{\omega}_{66}^{2}+\kappa^{2} \mu_{6}+\cdots
\end{aligned}
$$

Retaining the first term of the development (2.1), as applied to (4.5), untouched and ordering the rest of the terms according to ascending powers of $\kappa^{2}$, we may, after canceling the common factor $\omega^{2}-\omega_{33}^{2}$, insert successively the expressions (4.8) in (2.1) and thus determine the unknown coefficients $\mu_{1}, \cdots, \mu_{6}$. Thus we finally get the simple solution

$$
\begin{aligned}
& \mu_{1}=-\frac{\bar{\omega}_{15}^{2} \bar{\omega}_{51}^{2}}{\omega_{55}^{* 2}}, \quad \mu_{2}=-\frac{\bar{\omega}_{24}^{2} \bar{\omega}_{42}^{2}}{\omega_{44}^{* 2}}, \\
& \mu_{4}=\frac{\bar{\omega}_{46}^{2} \bar{\omega}_{64}^{2}}{\omega_{44}^{* 2}}, \quad \mu_{5}=\frac{\bar{\omega}_{56}^{2} \bar{\omega}_{65}^{2}}{\omega_{55}^{* 2}}, \\
& \mu_{6}=-\frac{\bar{\omega}_{46}^{2} \bar{\omega}_{84}^{2} \omega_{55}^{* 2}+\bar{\omega}_{55}^{2} \bar{\omega}_{85}^{2} \omega_{44}^{* 2}}{\omega_{44}^{* 2} \omega_{55}^{* 2}} .
\end{aligned}
$$




\section{Conclusions}

By using an expansion of the type (2.1) of the determinantal frequency equation (1.3), obtained for ordinary mechanical systems with $n$ degrees of freedom, it is easy to obtain approximate expressions (3.1), supposing "weak coupling" and distinct eigenvalues. By examples it is shown, that similar developments of the eigenvalues as (3.1) will hold also in cases, where at least some of the terms in the principal diagonal of the matrix are of the same order as the coupling terms.

\section{REFERENCES}

1. B. v. Schlippe, Jahrb. d. Luftfahrtforsch. 2, Triebwerk, 103-106 (1937).

2. K. Lürenbaum and W. Behrmann, loc. cit., 107-116 (1937).

3. M. Julien and Y. Rocard, Mécanique 23, 101-103 (1939).

4. R. C. Lewis and K. Unholtz, Refrig. Engn. 53, 291-295 (1947).

5. C. W. Kosten, On the elastic properties of vulcanized rubber (in Dutch), Dissertation, Delft (1942).

6. J. A. Haringx, On highly compressible helical springs and rubber rods, and their application for vibrationfree mountings (in Dutch), Dissertation, Delft (1947); see also Philips Research Reports 3, 401-449 (1948); 4, 49-80, 206-220, 261-290, 375-400, 407-448 (1949).

\section{BOOK REVIEWS}

Electromagnetic problems of microwave theory. By H. Motz. Methuen \& Co. Ltd., London, and John Wiley \& Sons, Inc., New York, 1951. vii + 184 pp. \$2.00.

This book is a new Methuen Monograph dealing in Chapter one with a summary of general topics in microwave methods including velocity modulated tubes, travelling wave tubes, resonators, cavity magnetrons, methods of detection, wavemeters, standing wave meters, the Smith chart, and matching. Chapters two and three deal with a detailed discussion of velocity modulation and Klystron theory following the work of Webster. Chapter four is entitled Mode Selection in Cavity Magnetrons and includes the Fourier Analysis of the rotating waves and conditions for synchronism of the electrons with the rotating field components. Some discussion of mode stability is given together with an estimate of magnetron efficiency.

Chapter five discusses the field relations in wave guides in orthogonal curvilinear systems. Application is made to guides of rectangular and circular cross section. The use of series expansions in terms of normal mode solutions is illustrated. The transmission line analogy is discussed briefly.

Calculation of electromagnetic fields in cavities and guides of complex shape is discussed in some detail in chapter six where relaxation and finite difference methods are used. The methods are illustrated with resonator gaps. The determination of the field pattern for higher modes is also discussed briefly. The analytical treatment of corrugated wave guides, as done by Wilkinshaw, is outlined very well in the space of about six pages.

Chapter seven is concerned with the impedance of an antenna in a wave guide. The problem of coupling into wave guides by means of straight wires or loops, as treated by the Toronto group, is presented in a very satisfactory way. The theory of discontinuities in wave guides as worked out by Bethe and Schwinger is outlined and applied to transverse windows, changes in cross section, and bends. The methods are applicable only to thin windows and sudden changes in cross section but a numerical method, applicable to any type of discontinuity, is outlined.

A large amount of useful material is packed into the 180 pages that make up this monograph. The writer did a very good job of discussing the problems which he includes. A person actively working in microwave theory will probably find this book useful as well as the person looking for general information. 